\title{
Facility Layout Planning with Continuous Rep- resentation Considering Temporal Efficiency*
}

\author{
Eiji Morinaga ${ }^{\dagger}$, Yutaro Shintome ${ }^{\dagger \ddagger}$, Hidefumi Wakamatsu ${ }^{\dagger}$ and Eiji Arai ${ }^{\dagger}$
}

\begin{abstract}
Facility layout planning (FLP) is one of the most important stages in the design of manufacturing systems. A major approach is to define an evaluation index based on distance and find a layout which minimizes it. In this approach, temporal efficiency is not considered in this stage but in the stage of production scheduling performed after completing FLP. The resultant temporal efficiency may not be optimal enough, since the scheduling is performed under the fixed layout. From this point of view, integration of FLP and production scheduling have been discussed in some works. However, detailed position and size of facilities were not considered. This paper provides an integrated method using continuous representation by which those factors can be dealt with. The problems of FLP and production scheduling are formulated as a mixed integer programming and a 0-1 integer programming, respectively. By assuming that transportation speed is constant, these problems are correlated by an equation. This correlation enables formulation of FLP considering temporal efficiency as a mixed integer programming. This method was applied to an example, and it was shown that facilities which are significant for production scheduling are located close enough and better temporal efficiency can be achieved.
\end{abstract}

\section{Introduction}

In order to convert data of an artifact created in product design into an entity and provide it to a customer as a product with high cost-performance, it is necessary to optimally design, properly manage and efficiently operate a manufacturing system. Facility layout planning (FLP) is one of the most important stages for the optimal design of a manufacturing system and has been a topic of discussion for a long time[1-3]. In FLP research, evaluation indices based on distance such as total travel distance and total material handling cost are usually defined, and optimization based on them is performed by mathematical optimization (quadratic assignment problem (QAP)[4] and mixed integer programming (MIP)[5]) or metaheuristics (tabu search[6], simulated annealing[7], genetic algorithm[8,9], etc.). Those indices do not include temporal efficiency and it is considered in the stage of production scheduling[10-12] performed after completing the FLP stage. In other words, optimiza-

\footnotetext{
* Manuscript Received Date: October 19, 2015

The material of this paper was partially presented at International Symposium on Flexible Automation 2014 (ISFA 2014) which was held in July, 2014.

$\dagger$ Division of Materials and Manufacturing Science, Osaka University; 2-1 Yamadaoka, Suita city, Osaka 565-0871, JAPAN

‡ Currently, Panasonic Welding Systems, Co. Ltd.
}

Key Words: facility layout planning, production scheduling, makespan, mixed integer programming. tion is independently performed in each of the two stages. However, this may result in inadequate optimization result from the point of view of the whole system. For example, optimization in terms of total travel distance may cause locating some facilities unnecessarily closer than they are required from the scheduling point of view and therefore other facilities which are required to be located as close as possible are located apart. Therefore, it is necessary to take temporal efficiency into account in FLP stage.

There are two reasons of performing the independent optimization. One is that a production schedule is prerequisite for evaluating temporal efficiency and therefore this evaluation cannot be performed in FLP stage. The other is that, unlike a production schedule, it is difficult to change a facility layout depending on changes in manufacturing situations such as demand fluctuation. For these decades in which manufacturing style has transitioned from low-mix high-volume manufacturing to high-mix low-volume manufacturing, flexibility and robustness of manufacturing systems have been a key concept. These concepts have driven development of methodologies of robust FLP $[13,14]$ where an optimal facility layout considering demand uncertainty is designed based on the indices that do not include temporal efficiency, and robust production scheduling[15] where an optimal schedule considering uncertainty in processing times, demand, etc. is discussed. These methodologies would provide solutions for the problem concern- 
ing to the latter reason.

This research aims to develop a methodology for FLP considering temporal efficiency which overcomes both of the above two problems. As the first step, this paper focuses on the problem concerning to the former reason and deals with FLP considering temporal efficiency for fixed manufacturing conditions. For this issue, several methods have been proposed [16-18]. However, they are for allocation of facilities to pregiven sites and detailed position and size of facilities are not taken into consideration. This paper provides a method for FLP with continuous representation, by which those factors are dealt with, considering temporal efficiency. In the next section, problems of FLP and production scheduling are formulated as an MIP and a 0-1 integer programming, respectively. In section 3., those two problems are then merged by focusing on required times for transportation between two facilities, and eventually FLP considering temporal efficiency is formulated as an MIP problem. This method is applied to an example and its effectiveness is evaluated in section 4.. Section 5. provides conclusions.

\section{Mathematical Formulation of FLP and Production Scheduling}

This research deals with job-shop production with $J$ jobs and $M$ machines. Job $j \in \mathcal{J} \equiv\{1, \ldots, J\}$ needs $O_{j}$ operations. Operation $o \in \mathcal{O}_{j} \equiv\left\{1, \ldots, O_{j}\right\}$ of job $j$ is processed by machine $\bar{m}_{j o} \in \mathcal{M} \equiv\{1, \ldots, M\}$. Width and depth of machine $m$ are $w_{m}$ and $d_{m}$. This section provides mathematical formulations of FLP and production scheduling, from which formulae of FLP considering temporal efficiency is derived in section 3.

\subsection{Formulation of FLP}

There are two well-known ways of formulating FLP problem. One is formulation as a QAP and the other is as an MIP. In QAP formulation, the production area is divided into a set of cells and each machine is assigned to one of those cells. Therefore, it is unsuitable for FLP dealing with detailed position of unequal-sized machines. For this reason, this paper adopts the MIP formulation. Positive continuous decision variables $x_{m}, y_{m}$ and 0 -1 decision variables $s_{m}$ are introduced, which stand for centroid and orientation of machine $m$, respectively. The FLP problem is formulated as an MIP as follows ${ }^{1}$ :

$$
\sum_{j=1}^{\operatorname{minimize}} \sum_{l=1}^{M} \sum_{m=1}^{M} f_{j l m}\left(X_{l m}+Y_{l m}\right)
$$

${ }^{1}$ This formulation is not a strict MIP, since equations (1) and (4) include absolute value functions and "max" functions. However, it can be equivalently transformed into a strict MIP by introducing artificial variables and using the "big-M" method.

$$
\begin{aligned}
& \text { subject to: } \\
& \frac{w_{m} s_{m}+d_{m}\left(1-s_{m}\right)}{2} \\
& \quad \leq x_{m} \leq W-\frac{w_{m} s_{m}+d_{m}\left(1-s_{m}\right)}{2}, \forall m \in \mathcal{M} \\
& \frac{d_{m} s_{m}+w_{m}\left(1-s_{m}\right)}{2} \\
& \quad \leq y_{m} \leq D-\frac{d_{m} s_{m}+w_{m}\left(1-s_{m}\right)}{2}, \forall m \in \mathcal{M} \\
& \max \left\{X_{l m}-\frac{w_{l} s_{l}+d_{l}\left(1-s_{l}\right)+w_{m} s_{m}+d_{m}\left(1-s_{m}\right)}{2},\right. \\
& \left.\quad Y_{l m} \geq \frac{d_{l} s_{l}+w_{l}\left(1-s_{l}\right)+d_{m} s_{m}+w_{m}\left(1-s_{m}\right)}{2}\right\} \geq 0,
\end{aligned}
$$

where $X_{l m}$ and $Y_{l m}$ are defined by $\left|x_{l}-x_{m}\right|$ and $\mid y_{l}-$ $y_{m} \mid$, respectively, and $f_{j l m}$ is the quantity of material $j$ transported from machine $l$ to $m$. $W$ and $D$ are the width and depth of the production area. Equation (1) describes the total travel distance for the production based on the Manhattan distance. Inequalities (2) and (3) assure every machine is included in the area, and (4) assures no machines overlap each other.

\subsection{Formulation of Production Schedul- ing}

In this paper, time is discretized with unit time $u$ and the planning period is composed of $T$ periods whose length is $u$. 0-1 decision variables $z_{\text {jomt }}$ are introduced for describing a production schedule mathematically. This variable takes 1 if operation $o$ of job $j$ is processed by machine $m$ at period $t \in \mathcal{T} \equiv\{1, \ldots, T\}$. The production scheduling problem for achieving the minimum makespan is formulated as a $0-1$ integer programming as follows ${ }^{2}$ :

$$
\max _{j \in \mathcal{J}}\left\{\frac{1}{\tau_{j O_{j}}} \sum_{t=1}^{T}\left(t-\frac{1}{2}\right) z_{j O_{j} \bar{m}_{j O_{j}} t}+\frac{\tau_{j O_{j}}}{2}\right\}
$$

subject to:

$$
\begin{aligned}
& \sum_{m=1}^{M} \sum_{t=1}^{T} z_{j o m t}=\tau_{j o}, \forall j \in \mathcal{J}, \forall o \in \mathcal{O}_{j} \\
& \sum_{j=1}^{J} \sum_{o=1}^{O_{j}} z_{j o m t} \leq 1, \forall m \in \mathcal{M}, \forall t \in \mathcal{T} \\
& \frac{1}{\tau_{j(o+1)}} \sum_{m=1}^{M} \sum_{t=1}^{T}\left(t-\frac{1}{2}\right) z_{j(o+1) m t}-\frac{\tau_{j(o+1)}}{2}+1 \\
& >\frac{1}{\tau_{j o}} \sum_{m=1}^{M} \sum_{t=1}^{T}\left(t-\frac{1}{2}\right) z_{j o m t}+\frac{\tau_{j o}}{2}+h_{j o}, \\
& \forall j \in \mathcal{J}, \forall o \in\left\{1, \ldots, O_{j}-1\right\}
\end{aligned}
$$

\footnotetext{
${ }^{2}$ This formulation is not a strict integer programming, since equation (5) includes a "max" function. It is transformed into a strict integer programming equivalently by introducing artificial variables.
} 


$$
\begin{aligned}
& \tau_{\text {jo }}\left(z_{\text {jom }(t+1)}-z_{\text {jomt }}\right) \leq \sum_{l=t}^{t+\tau_{j o}} z_{\text {joml }}, \\
& \quad \forall j \in \mathcal{J}, \forall o \in \mathcal{O}_{j}, \forall m \in \mathcal{M}, \forall t \in\left\{1, \ldots, T-\tau_{j o}\right\}(9) \\
& z_{\text {jomt }}=0, \forall m \neq \bar{m}_{j o}, \forall j \in \mathcal{J}, \forall o \in \mathcal{O}_{j}, \forall t \in \mathcal{T}(10)
\end{aligned}
$$

where $\tau_{j o}$ is the required period for processing the operation $o$ of job $j, h_{j o}$ is required time for transporting the material of job $j$ to the machine which performs operation $(o+1)$ after completing operation $o$. Equation (5) describes maximum of finish period of the last operation of each job, and minimizing this value results in minimization of makespan of the schedule. Equation (6) assures processing period for each operation equals to the given required period. Inequality (7) constrains a machine from performing multiple operations at the same time. Inequality (8) is for operation order constraints and equation (9) avoids interruption of an operation.

\section{FLP Considering Temporal Effi- ciency}

FLP and production scheduling relate each other by transportation of materials. The FLP problem and the production scheduling problem given in the previous section can be merged by formulating relationship between position of machines and transportation times. By assuming that the transportation speed $v$ is constant and by ignoring transportation routes and collisions, the relationship can be described by the following equation.

$$
h_{j o}=\frac{\left|x_{\bar{m}_{j(o+1)}}-x_{\bar{m}_{j o}}\right|+\left|y_{\bar{m}_{j(o+1)}}-y_{\bar{m}_{j o}}\right|}{v u}
$$

By using this equation, FLP considering temporal efficiency can be formulated as the following optimization problem which can be equivalently transformed into an MIP by introducing artificial variables and using the "big-M" method.

minimize:

$$
\max _{j \in \mathcal{J}}\left\{\frac{1}{\tau_{j O_{j}}} \sum_{t=1}^{T}\left(t-\frac{1}{2}\right) z_{j O_{j} \bar{m}_{j O_{j}} t}+\frac{\tau_{j O_{j}}}{2}\right\}
$$

subject to:

$$
\begin{aligned}
& \frac{w_{m} s_{m}+d_{m}\left(1-s_{m}\right)}{2} \\
& \quad \leq x_{m} \leq W-\frac{w_{m} s_{m}+d_{m}\left(1-s_{m}\right)}{2}, \forall m \in \mathcal{M} \\
& \frac{d_{m} s_{m}+w_{m}\left(1-s_{m}\right)}{2} \\
& \quad \leq y_{m} \leq D-\frac{d_{m} s_{m}+w_{m}\left(1-s_{m}\right)}{2}, \forall m \in \mathcal{M} \\
& \max \left\{X_{l m}-\frac{w_{l} s_{l}+d_{l}\left(1-s_{l}\right)+w_{m} s_{m}+d_{m}\left(1-s_{m}\right)}{2},\right. \\
& \left.Y_{l m} \geq \frac{d_{l} s_{l}+w_{l}\left(1-s_{l}\right)+d_{m} s_{m}+w_{m}\left(1-s_{m}\right)}{2}\right\} \geq 0,
\end{aligned}
$$

$$
\begin{aligned}
& \sum_{m=1}^{M} \sum_{t=1}^{T} z_{j o m t}=\tau_{j o}, \forall j \in \mathcal{J}, \forall o \in \mathcal{O}_{j} \\
& \sum_{j=1}^{J} \sum_{o=1}^{O_{j}} z_{j o m t} \leq 1, \forall m \in \mathcal{M}, \forall t \in \mathcal{T} \\
& \frac{1}{\tau_{j(o+1)}} \sum_{m=1}^{M} \sum_{t=1}^{T}\left(t-\frac{1}{2}\right) z_{j(o+1) m t}-\frac{\tau_{j(o+1)}}{2}+1 \\
& >\frac{1}{\tau_{j o}} \sum_{m=1}^{M} \sum_{t=1}^{T}\left(t-\frac{1}{2}\right) z_{j o m t}+\frac{\tau_{j o}}{2}+h_{j o}, \\
& \tau_{j o}\left(z_{j o m}(t+1)-z_{j o m t}\right) \leq \sum_{l=t}^{t+\tau_{j o}} z_{j o m l}, \\
& \forall j \in \mathcal{J}, \forall o \in \mathcal{O}_{j}, \forall m \in \mathcal{M}, \forall t \in\left\{1, \ldots, T-\tau_{j o}\right\}(19) \\
& z_{j o m t}=0, \forall m \neq \bar{m}_{j o}, \forall j \in \mathcal{J}, \forall o \in \mathcal{O}_{j}, \forall t \in \mathcal{T} \\
& h_{j o}=\frac{\left|x_{\bar{m}_{j(o+1)}}-x_{\bar{m}_{j o}}\right|+\left|y_{\bar{m}_{j(o+1)}}-y_{\bar{m}_{j o}}\right|}{v u}
\end{aligned}
$$

\section{Numerical Example}

The proposed method was applied to an example where $W=45[\mathrm{~m}], D=45[\mathrm{~m}], J=5, O_{1}=O_{2}=\ldots=$ $O_{5}=7, M=7, u=0.5[\mathrm{~min}], T=60$ and $v=15[\mathrm{~m} / \mathrm{min}]$. Dimensions of the machines were given as shown in Table 1. The machine processing each operation was given as Table 2, and its required time and period were given as Table 3 .

Table 1 Dimensions of machines ([m])

\begin{tabular}{|c|c|c|c|c|c|c|c|}
\hline Machine $m$ & 1 & 2 & 3 & 4 & 5 & 6 & 7 \\
\hline Width $\left(w_{m}\right)$ & 2 & 3 & 4 & 3 & 5 & 4 & 2 \\
\hline Depth $\left(d_{m}\right)$ & 2 & 2 & 2 & 3 & 4 & 3 & 2 \\
\hline
\end{tabular}

Table 2 Machine sequence $\bar{m}_{j o}$

\begin{tabular}{|c|c|c|c|c|c|c|c|}
\hline \multirow{2}{*}{ Job $j$} & \multicolumn{7}{|c|}{ Operation $O$} \\
\cline { 2 - 8 } & 1 & 2 & 3 & 4 & 5 & 6 & 7 \\
\hline 1 & 3 & 1 & 2 & 6 & 7 & 4 & 5 \\
\hline 2 & 2 & 1 & 5 & 7 & 3 & 4 & 6 \\
\hline 3 & 3 & 4 & 2 & 7 & 5 & 6 & 1 \\
\hline 4 & 2 & 4 & 5 & 6 & 7 & 3 & 1 \\
\hline 5 & 3 & 1 & 5 & 6 & 7 & 4 & 2 \\
\hline
\end{tabular}

Table 3 Required processing time $([\min ])$ and periods $\tau_{j o}$

\begin{tabular}{|c|c|c|c|c|c|c|c|}
\hline \multirow{2}{*}{$\begin{array}{c}\text { Job } \\
j\end{array}$} & \multicolumn{7}{|c|}{ Operation $O$} \\
\cline { 2 - 8 } & 1 & 2 & 3 & 4 & 5 & 6 & 7 \\
\hline 1 & $2(4)$ & $3(6)$ & $2(4)$ & $3(6)$ & $2(4)$ & $4(8)$ & $3(6)$ \\
\hline 2 & $3(6)$ & $1(2)$ & $3(6)$ & $2(4)$ & $4(8)$ & $3(6)$ & $2(4)$ \\
\hline 3 & $1(2)$ & $2(4)$ & $3(6)$ & $2(4)$ & $2(4)$ & $1(2)$ & $3(6)$ \\
\hline 4 & $3(6)$ & $2(4)$ & $4(8)$ & $3(6)$ & $1(2)$ & $3(6)$ & $2(4)$ \\
\hline 5 & $2(4)$ & $1(2)$ & $4(8)$ & $3(6)$ & $1(2)$ & $3(6)$ & $2(4)$ \\
\hline
\end{tabular}

The optimizations were performed with a generic workstation (Dell Precision T7400, Intel Xeon E5430@ 


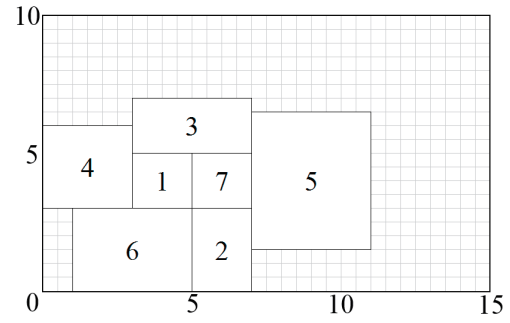

Fig. 1 Facility layout generated by the conventional method
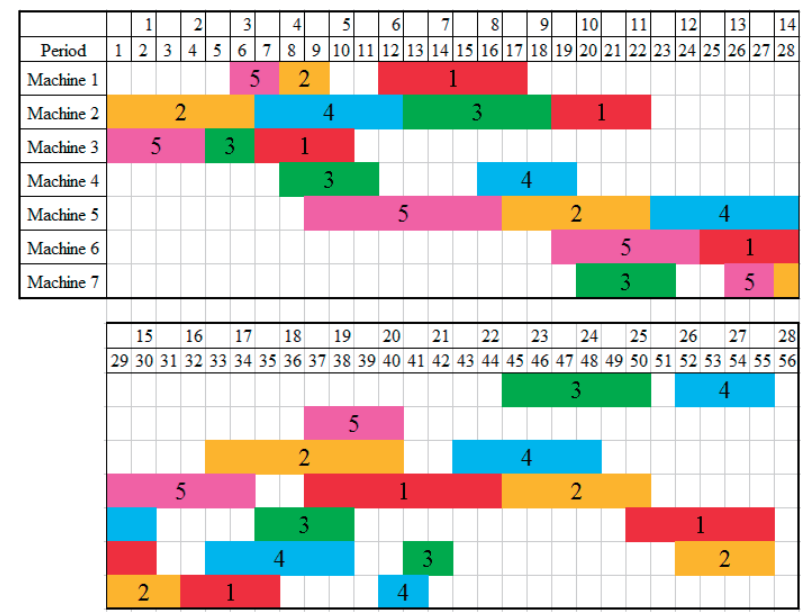

Fig. 2 Production schedule generated by the conventional method

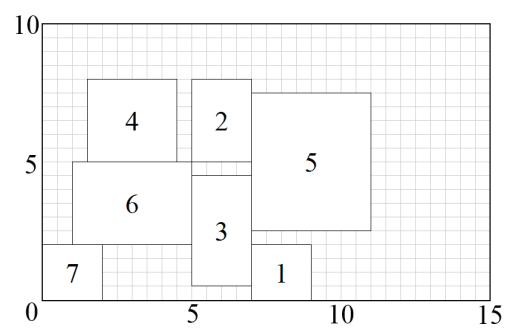

Fig. 3 Facility layout generated by the proposed method
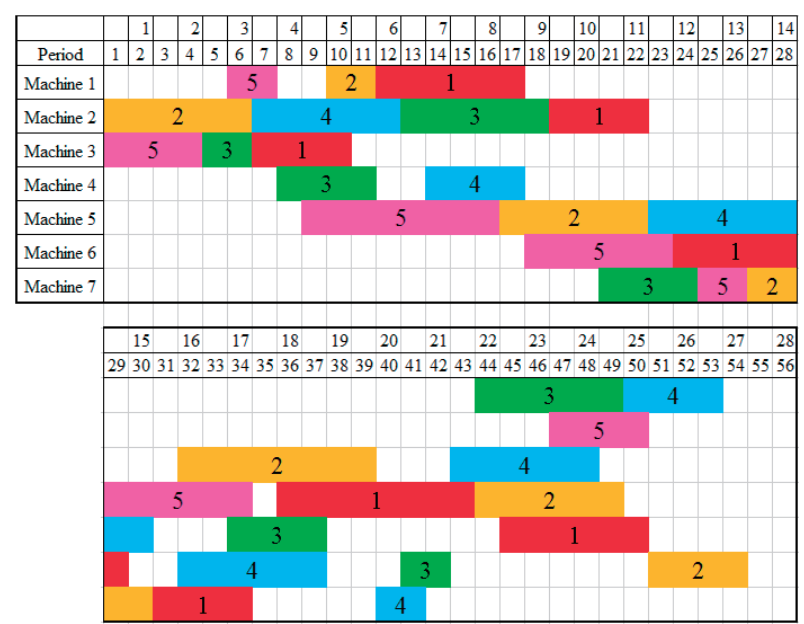

Fig. 4 Production schedule generated by the proposed method

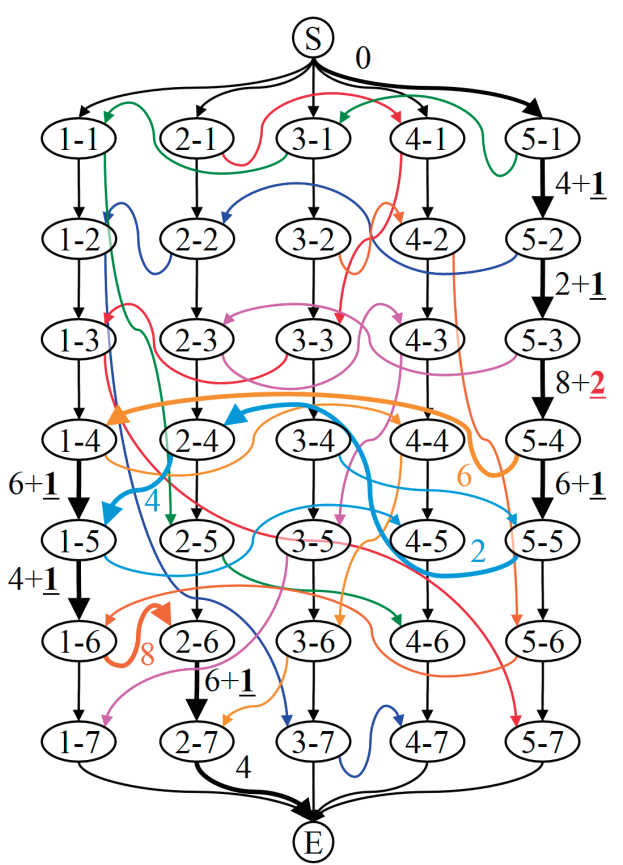

Fig. 5 Disjunctive graph of the schedule obtained by the conventional method. Thick arrows stand for the critical path. The values with no underline between two nodes on the critical path denote periods for processing the job and the underlined values denote periods for transporting the material.

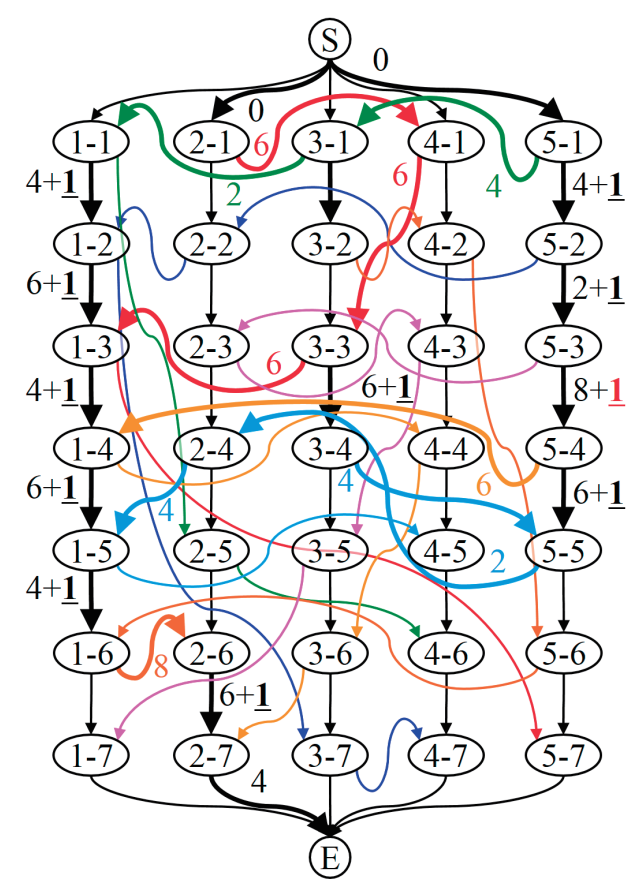

Fig. 6 Disjunctive graph of the schedule obtained by the proposed method. Thick arrows stand for the critical path. The values with no underline between two nodes on the critical path denote periods for processing the job and the underlined values denote periods for transporting the material. 
2.66GHz, 20GB RAM) and a commercial solver (ILOG CPLEX Optimization Studio 12.5). Figs. 1, 2 show the layout and the schedule obtained by the conventional method in which the two optimization problems in Section 2. are solved individually, and Figs. 3, 4 show those obtained by the proposed method given in Section 3., respectively. Figs. 5, 6 show disjunctive graphs of the two schedules. In these figures, thick arrows show the critical paths. The values without underlines beside those arrows are required periods for performing the operations $\tau_{j o}$ given in Table 3 and those with underlines are for transporting the materials $\left\lceil h_{j o}\right\rceil$ ( $\lceil\cdot\rceil$ stands for the ceiling function) which are shown in detail in Tables 4, 5. In Fig. 5, required periods for transportation in the critical path was not minimized enough and it takes 2 periods to transport the material of job 5 from the 3rd operation (performed by machine 5) to the 4th operation (performed by machine 6). On the other hand, in Fig. 6, all the required periods for transportation in the critical path were minimized and the shorter makespan was achieved as a result.

Table 4 Required period for transportation $\left\lceil h_{j o}\right\rceil$ obtained by the conventional method

\begin{tabular}{|c|c|c|c|c|c|c|}
\hline Job & \multicolumn{7}{|c|}{ Operation $o$} \\
\cline { 2 - 7 }$j$ & $1 \rightarrow 2$ & $2 \rightarrow 3$ & $3 \rightarrow 4$ & $4 \rightarrow 5$ & $5 \rightarrow 6$ & $6 \rightarrow 7$ \\
\hline 1 & 1 & 1 & 1 & 1 & 1 & 2 \\
\hline 2 & 1 & 1 & 1 & 1 & 1 & 1 \\
\hline 3 & 1 & 1 & 1 & 1 & 2 & 1 \\
\hline 4 & 1 & 2 & 1 & 1 & 1 & 1 \\
\hline 5 & 1 & 1 & 2 & 1 & 1 & 1 \\
\hline
\end{tabular}

Table 5 Required period for transportation $\left\lceil h_{j o}\right\rceil$ obtained by the proposed method

\begin{tabular}{|c|c|c|c|c|c|c|}
\hline Job & \multicolumn{7}{|c|}{ Operation $o$} \\
\cline { 2 - 7 } & $1 \rightarrow 2$ & $2 \rightarrow 3$ & $3 \rightarrow 4$ & $4 \rightarrow 5$ & $5 \rightarrow 6$ & $6 \rightarrow 7$ \\
\hline 1 & 1 & 1 & 1 & 1 & 1 & 1 \\
\hline 2 & 1 & 1 & 2 & 1 & 1 & 1 \\
\hline 3 & 1 & 1 & 2 & 2 & 1 & 1 \\
\hline 4 & 1 & 1 & 1 & 1 & 1 & 1 \\
\hline 5 & 1 & 1 & 1 & 1 & 1 & 1 \\
\hline
\end{tabular}

\section{Conclusion}

In this paper, a method for FLP with continuous representation considering temporal efficiency has been presented. FLP has been formulated as an MIP, by which detailed position and size of facilities can be taken into consideration, and production scheduling as a $0-1$ integer programming, respectively. Assumption of constant transporting speed correlated those two problems, and then FLP considering temporal efficiency has been formulated as an MIP. An example showed that the proposed method generates the layout which includes information on detailed position and size of facilities, so that required transportation times in the critical path of the schedule are minimized enough and achieves better temporal efficiency than the conventional method in which the above two problems are solved individually.

Although the potential of the proposed method was proven, there are two problems to be resolved in future works. One is concerned with formulation. This method is based on the formulation of the production scheduling problem as a 0-1 integer programming. In this formulation, it is necessary to define a variable for each period in the planning period. Therefore, setting the unit time smaller and dividing the planning period into smaller periods for higher accuracy make the number of the variables huge, and it is impossible to solve the optimization problem, though it has been possible to solve a 0-1 integer programming problem in a reasonably short time because of recent advances in computer technology and solvers. This point also makes it impossible to apply the proposed method to actual manufacturing where a large number of jobs and operations are required. This problem may be resolved by applying another formulation of production scheduling such as all integer programming or MIP to this method. The other problem is concerned with definition of distance among facilities. In this paper, distance among facilities has been evaluated based on the Manhattan distance. This is not reasonable from the point of view of actual manufacturing where materials are transported on complicated routes, and distance should be evaluated considering traveling routes.

These two points are the reasons why the improvement of temporal efficiency in the example was small, and resolving these two problems in future works would prove effectiveness of the proposed method more apparently.

In addition, it is also necessary to enhance this method from the point of view of robustness for taking various situation into consideration and applying the method to actual manufacturing, as described in Section 1.. In robust FLP, the robustness of a layout is typically defined as deviation in the evaluation index for the optimal solution for each product demand scenario or as that the degree of deviation is within a prespecified percentage. These definition will be applied to the evaluation index of the proposed method and methods for solving the robust problem will be studied in future works.

\section{References}

[1] R. D. Meller and K. Y. Gau: The facility layout problem: Recent and emerging trends and perspectives; Journal of Manufacturing Science, Vol. 15, No. 5, pp. 351-366 (1996)

[2] S. Benjaafar, S. S. Heragu and S. A. Irani: Next generation factory layouts: Research challenges and recent progress; Interfaces, Vol. 32, pp. 58-76 (2002)

[3] A. Drira, H. Pierreval and S. Hajri-Gabouj: Facility layout problems: A survey; Annual Reviews in 


\section{Control, Vol. 31, pp. 255-267 (2007)}

[4] E. L. Lawler: The quadratic assignment problem; Management Science, Vol. 19, pp. 586-599 (1963)

[5] R. D. Meller, V. Narayanan and P. H. Vance: Optimal facility layout design; Operations Research Letters, Vol. 23, pp. 117-127 (1999)

[6] W. C. Cheng and P. Kouvelis: An improved tabu search heuristic for solving facility layout design problems; International Journal of Production Research, Vol. 34, Issue 9, pp. 2565-2585 (1996)

[7] L. Chwif, M. R. P. Barretto and L. A. Moscato: A solution to the facility layout problem using simulated annealing; Computers in Industry, Vol. 36, Issues 12, pp. 125-132 (1998)

[8] P. Banerjee and Y. Zhou: Facilities layout design optimization with single loop material flow path configuration; International Journal of Production Research, Vol. 33, Issue 1, pp. 183-203 (1995)

[9] C. Hicks: A genetic algorithm tool for designing manufacturing facilities in the capital goods industry; International Journal of Production Economics, Vol. 90, pp. 199-211 (2004)

[10] S. C. Graves: A review of production scheduling; $O p$ erations Research, Vol. 29, No. 4, pp. 646-675 (1981)

[11] T. C. E. Cheng and M. C. Gupta: Survey of scheduling research involving due date determination decisions; European Journal of Operational Research, Vol. 38, No. 2, pp. 156-166 (1989)

[12] D. Lei: Multi-objective production scheduling: A survey; International Journal of Advanced Manufacturing Technology, Vol. 43, Nos. 9-10, pp. 926-938 (2009)

[13] G. Aiello and M. Enea: Fuzzy approach to the robust facility layout in uncertain production environments; International Journal of Production Research, Vol. 39, pp. 4089-4101 (2001)

[14] G. Moslemipour, T. S. Lee and D. Rilling: A review of intelligent approaches for designing dynamic and robust layouts in flexible manufacturing systems; International Journal of Advanced Manufacturing Technology, Vol. 60, pp. 11-27 (2012)

[15] R. L. Daniels and P. Kouvelis: Robust scheduling to hedge against processing time uncertainty in singlestage production; Management Science, Vol. 41, pp. 363-376 (1995)

[16] Y. Fujihara and H. Osaki: A facility layout method linked to production scheduling; Transactions of the Japan Society of Mechanical Engineers, Series C, Vol. 63, pp. 293-303 (1997) (In Japanese)

[17] R. Hino and T. Moriwaki: Resource reallocation based on production scheduling (1st report); Journal of the Japan Society for Precision Engineering, Vol. 69, pp. 655-659 (2003) (In Japanese)

[18] T. Inagawa: Research on facility layout with production scheduling including transportation time; Master's Thesis, Hosei University (2005) (In Japanese)

\section{Authors}

Eiji Morinaga (Member)

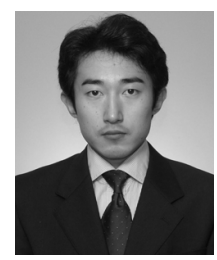

Eiji Morinaga received his B. Eng., M. Eng. and Ph.D. degrees in Mechanical Engineering from Osaka University, Japan, in 2000, 2002 and 2005, respectively. From 2005 to 2007, he worked as a Designated Researcher at Center for Advanced Science and Innovation, Osaka University. He then became an Assistant Professor at Division of Materials and Manufacturing Science, Osaka University. His research interests include system design and integration in product design and manufacturing. He is a member of JSME, JSPE, JWS, JIEP and TMSJ.

\section{Yutaro Shintome}

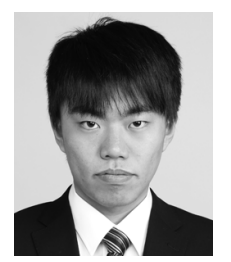

Yutaro Shintome received his B.Eng. and M.Eng. degrees from Osaka University, Japan, in 2012 and 2014, respectively. He then joined Panasonic Welding Systems, Co. Ltd.

\section{Hidefumi WaKamatsu (Member)}

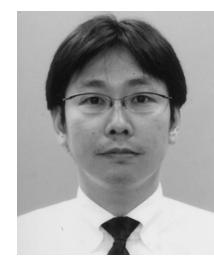

Hidefumi Wakamatsu received his B. Eng., M. Eng. and Ph. D. degrees from Osaka University, Japan, in 1993, 1994 and 2001, respectively. From 1995 to 2006, he worked as a Research Associate, Osaka University. He then became an Associate Professor at Division of Materials and Manufacturing Science, Osaka University. His research interests include handling of flexible object. He is a member of RSJ, JSME, JSPE, JWS and TMSJ.

\section{Eiji Arai (Member)}

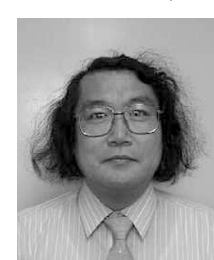

Eiji Arai received his B.Eng., M.Eng. and Dr.Eng. degrees from The University of Tokyo, Japan, in 1975, 1977 and 1980, respectively. He worked as a Research Associate at Kobe University from 1980 to 1984, an Associate Professor at Shizuoka University from 1984 to 1992, and an Associate Professor at Tokyo Metropolitan University from 1992 to 1995, respectively. He then joined Osaka University at 1995, where he is currently Professor of Division of Materials and Manufacturing Science. His research interests include intelligent $\mathrm{CAD} / \mathrm{CAM}$ for mechanical design. He is a member of JSME, JSPE, JWS, TMSJ, etc. 